\title{
Chiral Photonic Metamaterial
}

\author{
V. A. Fedotov ${ }^{1}$, E. Plum ${ }^{1, \#}$, Y. Chen ${ }^{2}$, A. S. Schwanecke ${ }^{1}$, and N. I. Zheludev ${ }^{1}$ \\ ${ }^{1}$ EPSRC NanoPhotonics Portfolio Centre, Optoelectronics Research Centre, University of Southampton, SO17 IBJ, UK \\ ${ }^{2}$ Central Microstructure Facility, Rutherford Appleton Laboratory, Oxfordshire, OX11 OQX, UK
}

\begin{abstract}
We demonstrate novel chiral photonic metamaterials consisting of physically separated mutually twisted planar metal patterns in parallel planes. Such metamaterials are shown to exhibit very strong gyrotropy $\left(600^{\circ} / \mathrm{mm}\right)$ in the visible, near-IR and microwaves.
\end{abstract}

Materials exhibiting strong gyrotropy were always of interest for various optoelectronic, life science microscopy and display applications but recently have started to attract a lot of attention as potential candidates for achieving negative refection [1]. However, gyrotropic response of most of the naturally available materials is rather weak and therefore artificially structured materials or, in other words, metamaterials have to be considered. Sculptured thin films in the form of helical pillars have been discussed as possible solutions for obtaining strong artificial gyrotropy in the optical part of the spectrum. From the metamaterial perspective, however, it is very desirable if gyrotropy can be achieved by planar patterning using well-established planar nano-technologies, which will make fabrication of optically active nano-structures a practical proposition. It has been suggested and recently demonstrated in the microwave part of the spectrum that electromagnetic coupling between layers of mutually twisted metal patterns can create $3 \mathrm{D}$ chirality showing strong gyrotropic response $[2,3]$.

Here, for the first time, we demonstrate a new type of chiral photonic metamaterial exhibiting strong gyrotropy in the optical and microwave part of the spectrum. It is a periodic bilayered structure, which consist of pairs of identical planar metal patterns located in parallel planes and mutually twisted by $15^{\circ}$ (see Fig. 1a).

The metamaterial for microwaves (see Fig. 1b) was made of pairs of copper rosettes edged on both sides of a standard $1.5 \mathrm{~mm}$ thick PC-board with the pitch of $15 \mathrm{~mm}$. For the optical part of the spectrum we manufactured and studied two versions of the metamaterial, one made of pairs of rosette-like nano-patterns (see Fig. 1c) and the other made of nano-patterns that resemble a cross. Both nano-patterns were edged from $50 \mathrm{~nm}$ thick Al layers and are periodically structured with a pitch of $700 \mathrm{~nm}$. The patterns in each pair were separated by $50 \mathrm{~nm}$ of dielectric layer making a chiral bilayered structure only $150 \mathrm{~nm}$ thick. For comparison we also studied two reference nanostructures with no structural twist formed by one and two layers of (perfectly aligned) Al rosettes respectively.
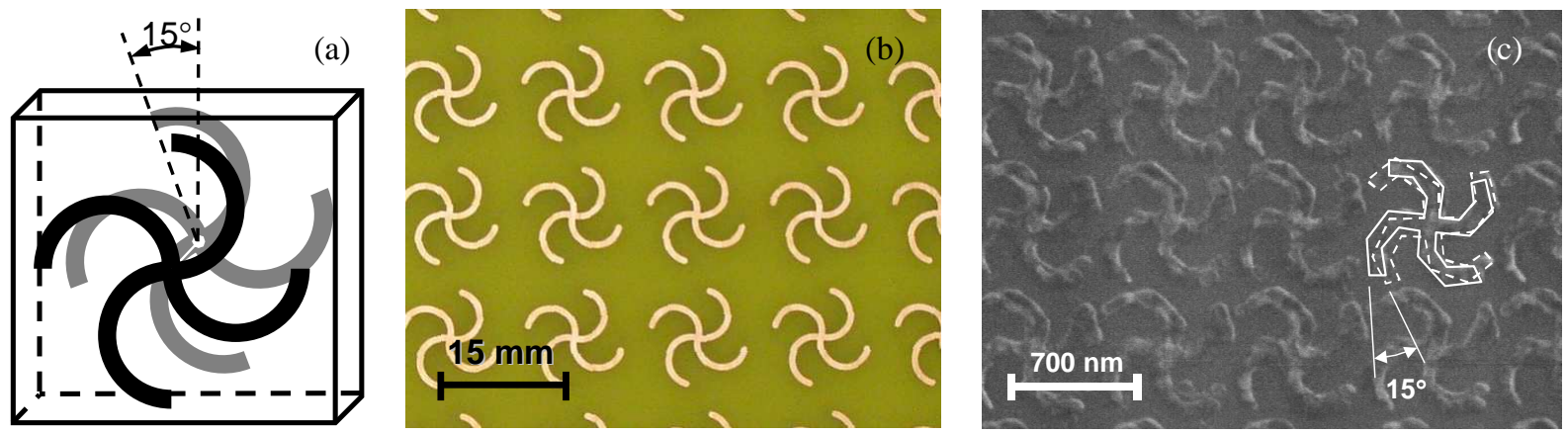

Fig. 1. (a) Schematics of the chiral bilayered metamaterial unit cell. (b) Microwave gyrotropic metamaterial consisting of twisted pairs of copper rosettes edged on both sides of a PC-board. (c) SEM micrograph of the gyrotropic metamaterial for the optical part of the spectrum, which consists of twisted pairs of $50 \mathrm{~nm}$ thick Al rosettes separated by only $50 \mathrm{~nm}$ of dielectric layer. 

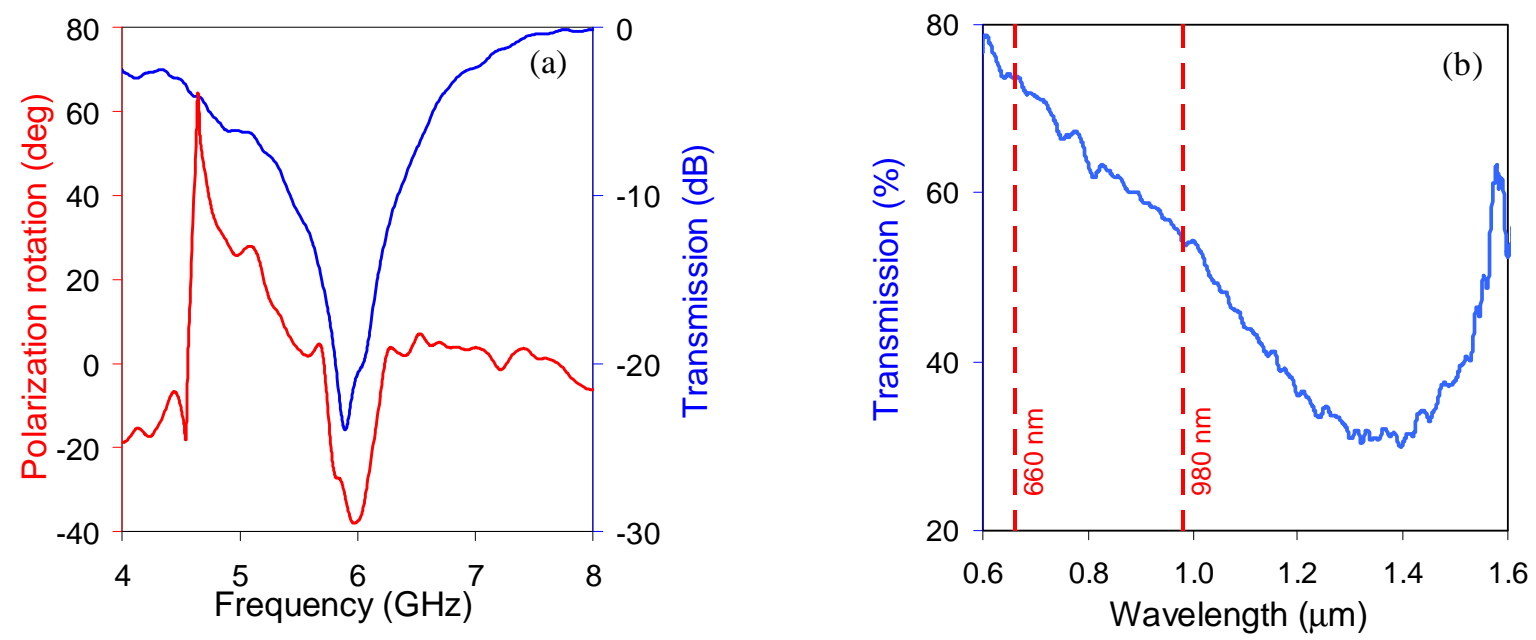

Fig. 2. (a) Rotation of the plane of polarization and transmission by the microwave-metamaterial. (b) Transmission resonance of the twisted rosettes nano-metamaterial. The wavelengths for which the polarization rotation has been measured are marked.

We have conducted measurements of the polarization rotation and transmission of the microwave metamaterial the results of which are presented in Fig. $2 \mathrm{a}$ for the $4-8 \mathrm{GHz}$ range. Both polarization rotation and transmission show a resonance at about $6 \mathrm{GHz}$ (see Fig. 2a). At this resonance transmission is a minimum while the gyrotropy becomes exceptionally strong, exceeding a polarization rotation of $40^{\circ}$. This is remarkably strong, considering that the material is 30 times thinner than the wavelength.

The optical activity of the nano-structured metamaterials was measured at $660 \mathrm{~nm}$ and $980 \mathrm{~nm}$. The metamaterials that consisted of two mutually twisted elements clearly showed optical activity at both wavelengths. For "twisted rosettes" we measured polarization rotation of a linearly polarized laser beam of about $0.09^{\circ}$ at 660 and $980 \mathrm{~nm}$, while "twisted crosses" exhibited polarization azimuth rotation of about $0.05^{\circ}$ at both wavelengths. In terms of specific optical activity the effect in the "twisted crosses" nano-structure exceeds $300 \% \mathrm{~mm}$, while the "twisted rosettes" nano-structure shows as much as $600 \% \mathrm{~mm}$. Furthermore, based on our microwave measurements we expect resonant increase of optical activity at wavelengths close to each metamaterial's transmission resonance, which, in case of twisted rosettes, is located at around $1.4 \mu \mathrm{m}$ (see Fig. 2b).

In conclusion, we have demonstrated that arrays of pairs of mutually twisted planar metal patterns if physically separated can form photonic media that are highly gyrotropic in the microwave and optical part of the spectrum. We found that optical activity of the chiral layered nano-structures can reach $600 \% \mathrm{~mm}$ in the visible-to-near IR part of the spectrum $(660-980 \mathrm{~nm})$. At $980 \mathrm{~nm}$ this is about two orders of magnitude stronger than the optical activity of quartz. We expect even higher values of optical activity at longer wavelengths close to the metamaterials' transmission resonances. This fuels hopes that chirality driven negative refraction can be observed in the optical part of the spectrum using the proposed gyrotropic layered metamaterials. Importantly, such media can be produced using well-established planar patterning technologies, which makes them much easier and cheaper to fabricate than non-planar chiral structures that have been suggested in the literature.

[1] J. B. Pendry, “A Chiral Route to Negative Refraction,” Science 306, 1353 (2004).

[2] Y. Svirko, N. Zheludev, and M. Osipov, "Layered chiral metallic microstructures with inductive coupling," Appl. Phys. Lett. 78, 498 (2001).

[3] A. V. Rogacheva, V. A. Fedotov, A. S. Schwanecke, and N. I. Zheludev, "Giant Gyrotropy due to Electromagnetic-Field Coupling in a Bilayered Chiral Structure," Phys. Rev. Lett. 97, 177401 (2006). 